\title{
Adaptability Analysis of 6LoWPAN and RPL for Healthcare applications of Internet-of-Things
}

\author{
Jayaram Hariharakrishnan ${ }^{1}$, N Bhalaji ${ }^{1}$ \\ Department of Information Technology, SSN College of Engineering \\ bhalajin@ssn.edu.in
}

\begin{abstract}
Ubiquitous Networks powered by Wireless Sensor Networks (WSN) is cutting across many technologies assisting day-to-day human activities. This technology confers the ability to sense and surmise the external environmental factors of various ecologies. Interconnection of these sensing devices for Machine to Machine (M2M) communication leads to the origination of Internet-of-Things (IoT). Recent advancements in the technology of Internet-of-Things guides the production of smart objects that can accomplish location, identification, connection and measurement of external factors. This leads to a new type of communication paradigm between objects and humans. One of the important problem due to the population explosion that can be addressed by IoT is the Healthcare of individual human beings. Remote health monitoring is one of the greatest technology exploited in medical professionals to keep a check on the patient's important health factors periodically. This was done in a smaller geographical area before the era of IoT. As IoT can communicate to other Internet, This remote healthcare monitoring can now be applied over a wider geographical topology. This paper extensively analyses the performance of 6LoWPAN and RPL IoT for healthcare applications. This paper especially focuses on monitoring an athlete's thermoregulation. Also, a new technique to identify and train marathon athletes to the race topography has been proposed. In this technique, each athlete is fitted with wearable sensors in their body in the training session to monitor and analyze the thermoregulation process occurring during training. After a detailed analysis of the athletes' thermoregulation process, personal training schedules are charted down according to variation in the thermoregulation process in each athlete. This technique helps to monitor each athlete's progress personally with less number of coaches and medical professionals leading to the prevention of unexpected death of a healthy athlete.
\end{abstract}


Journal of ISMAC (2021)

Vol.03/ No.02

Pages: 69-81

http://irojournals.com/iroismac/

DOI: https://doi.org/10.36548/jismac.2021.2.001

Keywords: Thermoregulation, RPL, 6LOWpan, IOT-Healthcare.

\section{MOTIVATION AND OBJECTIVE}

Unexpected death happens to marathon athletes' due to various external and internal factor. Such incidents in recent times are the main driving factor behind this paper. In 2013, One athlete dead and many were hospitalized due to hyperthermia after crossing the finish line [1]. An athlete after finishing the London Marathon in 2014 collapsed and died after being hospitalized [2]. In the same way, a 23-year-old athlete dies in a Pennsylvania half marathon due to hyperthermia after crossing the finish line [3]. Even though these athletes strive to maintain their fitness, these unexpected incidents bring sorrow to the entire sporting world. Many researchers focus on providing healthcare solutions to aged people. This project aims to avoid these unexpected incidents by monitoring the athletes' thermoregulation process with the help of IoT during training and charting down separate training schedules for each athlete according to their thermoregulatory conditions. Lack of effective training for marathon athletes' is considered as one of the major factors for this unexpected death of healthy athletes'. The main objective of the project is to develop a new architecture to monitor athletes' thermoregulation by fitting sensors to their body during training. Each temperature sensor sends the temperature information of athletes' to a centralized server that can be revisited by a coach or medical personnel's to assess the athletes' training report. As each athlete varies on different attributes, each athlete is given a separate training schedule according to their training report and can achieve the desired thermoregulatory conditions sooner than the results achieved in conventional group training. Also, we analyse the performance of two standardised low power protocols 6LoWPAN and RPL for the above-mentioned application.

\section{INTERNET-OF-THINGS}

Internet-of-Things (IoT) is an emerging area of research being unveiled by many researchers around the globe because of its immense applications in all aspects especially in assisting dayto-day human life. This is strongly associated with

- Ubiquitous computing,

- $\quad$ pervasive computing and 
- Ambient intelligence [4,5].

From the above definition, the following can be claimed as archetypal characteristics of IoT

- $\quad$ IoT is an independent network that operates on its infrastructures.

- $\quad$ IoT can be implemented for new services especially to assist human life.

- $\quad$ IoT enables new communication paradigms like Human-SO and S0-SO.

SO's are the building blocks of IoT. SO's should possess four common technical attributes

- $\quad$ Naming schemes

- $\quad$ Locating Schemes

- $\quad$ Sensing abilities

- $\quad$ Communicating abilities

IoT will be a gap bridging technology between the real world and the Internet by furnishing pertinent information at the right time [6]. In future, IoT will play an important part in Internet Services.

\section{THERMOREGULATION IN ATHLETES'}

Homeotherms are the species that maintains the narrow operative range of internal body temperatures (core temperature, Tc) despite exposure to varying environmental extremes [7]. In humans, this task is credibly accomplished by thermoregulatory systems using sensory feedback to the hypothalamus from thermoreceptors within the peripheral and central nervous system [8]. The neural autonomic outflow is stimulated by small variations in Tc. This ensures that a series of reflex movements adjust Tc by heat loss or production to respond to the change in body temperature. Measurement of rectal temperature (Tre) is the most commonly used technique to study the impact of exercise on Tc. Many factors can influence Tre [9] but no scientific findings have proved certain individual or group of factors has the most impact during marathon race-like circumstances. Environmental conditions [10-14], dehydration [15] and metabolic rate are commonly referenced as limiting factors for thermoregulatory control. However, gender may also contribute to other factors. Table 1 gives brief literature on distance running activities of athletes denoting the distance, number and gender of athletes', the 
environment of the race topography, Dehydration percentage of the athletes', rectal temperature and speed they ran during the activity.

\begin{tabular}{|l|r|l|l|r|r|r|}
\hline Reference & Distance & Participan & Environmentt [Tdb,re & DEH (\%) & Tre & \multicolumn{1}{l|}{ Speed } \\
\hline & $(\mathrm{km})$ & $\mathrm{(n})$ & & & $\left({ }^{\circ} \mathrm{C}\right)$ & $(\mathrm{m} / \mathrm{min})$ \\
\hline$[15]$ & 42.2 & $\mathrm{M} 1$ & $10^{\circ} \mathrm{C}$ & 2.13 & 39.4 & 256 \\
\hline$[16]$ & 42.2 & $\mathrm{M} 1$ & $23.9-27.8^{\circ} \mathrm{C}$ & 8.1 & 38.5 & 314 \\
\hline$[17]$ & 42.2 & $\mathrm{M} 1$ & $25.6^{\circ} \mathrm{C}, 35 \%$ & 5.1 & 37.3 & 302 \\
\hline$[18]$ & 42.2 & $\mathrm{M} 4$ & $20.4^{\circ} \mathrm{C}, 37 \%$ & 6.4 & 39 & 266 \\
\hline$[19]$ & 42.2 & $\mathrm{M} 8 ; \mathrm{F} 3$ & $16.1-5^{\circ} \mathrm{C}, 60-67 \%$ & 6.6 & 39.1 & 216 \\
\hline$[20]$ & 42.2 & $\mathrm{~F} 1$ & $18.1-20.5^{\circ} \mathrm{C}$ & 4.6 & 39 & 180 \\
\hline$[21]$ & 42.2 & $\mathrm{M} 18$ & $13.1-13.7^{\circ} \mathrm{C}, 99-100 \%$ & 2.9 & 40 & 203 \\
\hline$[22]$ & 42.2 & $\mathrm{M} 9$ & $21.7^{\circ} \mathrm{C}, 69 \%$ & 3.3 & 39.1 & 199 \\
\hline$[23]$ & 42.2 & $\mathrm{M} 7$ & $26^{\circ} \mathrm{C}$ & 3.5 & 36.5 & 142 \\
\hline$[24]$ & 42.2 & $\mathrm{M} 6$ & $21-26^{\circ} \mathrm{C}, 50-60 \%$ & 3.7 & 38.5 & 194 \\
\hline$[25]$ & 42.2 & $\mathrm{M} 2$ & $18.6^{\circ} \mathrm{C}$ & 2.9 & 40.5 & 259 \\
\hline$[26]$ & 42.2 & $\mathrm{M} 2$ & $17.9^{\circ} \mathrm{C}$ & 3.1 & 39.9 & 268 \\
\hline$[27]$ & 42.2 & $\mathrm{M} 6$ & $15-29^{\circ} \mathrm{C}$ & 4.3 & 39.8 & 257 \\
\hline$[28]$ & 42.2 & $\mathrm{M} 59$ & $10.8-12.1^{\circ} \mathrm{C}, 70-80 \%$ & 2.8 & 38.8 & 191 \\
\hline$[29]$ & 42.2 & $\mathrm{M} 62$ & $6.6-11.8^{\circ} \mathrm{C}$ & 3.2 & 38.7 & 198 \\
\hline$[30]$ & 42.2 & $\mathrm{M} 47$ & $10-12^{\circ} \mathrm{C}$ & 3 & 38.2 & 194 \\
\hline$[31]$ & 42.2 & $\mathrm{M} 20$ & $13.7-16^{\circ} \mathrm{C}$ & 4.97 & 36.5 & 303 \\
\hline$[32]$ & 42.2 & $\mathrm{M} 6$ & $17.5-20.4^{\circ} \mathrm{C}$ & 3.2 & 39.5 & 194 \\
\hline$[33]$ & 42.2 & $\mathrm{M} 3$ & $15.5-24.5^{\circ} \mathrm{C}$ & 4.7 & 39.2 & 194 \\
\hline
\end{tabular}

\section{TABLE 1. LITERATURE OF DISTANCE RUNNING ACTIVITIES}

\section{RPL: IPV6 ROUTING PROTOCOL FOR LOW POWER AND LOSSY NETWORKS}

The Internet Engineering Task Force in 2008 recognised the need for standardising the routing protocol for IPv6 smart objects and formed the working group Routing for Low powered and Lossy networks (ROLL). They standardised the RPL protocol in the year 2008 [34,35].

LLN's are networks with very limited energy, computation and bandwidth making them highly exposed to packet losses. RPL addresses two main problems

(i) Communication over a low data rate

(ii) Communication prone to high Bit Error Rate (BER)

A Lossy link is a link with high BER and long inaccessibility time. This strongly influences the design of the routing protocol. RPL is highly adaptive to varying network conditions by providing alternate routes if default routes are inaccessible [34]. 
Journal of ISMAC (2021)

Vol.03/ No.02

Pages: 69-81

http://irojournals.com/iroismac/

DOI: https://doi.org/10.36548/jismac.2021.2.001

\section{RPL Operation Overview}

DODAG built by RPL is a logical topology built over a physical network to meet specific criteria. Also, a network administrator may configure the network to have multiple routing topologies active in parallel to carry traffic with a different set of requirements. A node in the network can participate and join one or more DODAG [34].

\section{Formation of DODAG}

A node in the graph is configured as a root or LoWPAN Border Router (LBR) by the system administrator. Multiple nodes can be configured as root in the system. RPL specifies a new set of ICMPv6 control messages to exchange graph-related attributes[36]. The messages are

- DIS (DODAG Information Solicitation)

- $\quad$ DIO (DODAG Information Object) and

- $\quad$ DAO (DODAG Destination Advertisement Object).

RPL also supports point-to-point $(\mathrm{P} 2 \mathrm{P})$ data communication from one node to any other node in the DODAG. If a node sends the packet to another node within the LLN network, the packet first reaches 'up' to a common ancestor and then forwarded in the 'down' direction to the destination [37-39].

\section{(i) Global Repair and Local Repair}

A key feature of any routing protocol is the ability to repair the routing topology when failures occur. Likewise, RPL also supports topology repair mechanisms in case of link or node failures. Extreme care must be taken to avoid triggering a repair mechanism in transient conditions. RPL specifies two techniques known as local and global repair. If a link or neighbouring node is unavailable and has no other route in the 'up' direction, a local repair is triggered to quickly find an alternate parent/path. This is a local repair having no global implication [40]. Global repair is a mechanism that rebuilds the graph again at a cost. This can be triggered only from the root and has the cost of additional control traffic in the network. Each node in the graph will rerun the objective function for preferred parent selection [39, 40]. 
Journal of ISMAC (2021)

Vol.03/ No.02

Pages: 69-81

http://irojournals.com/iroismac/

DOI: https://doi.org/10.36548/jismac.2021.2.001

\section{(ii) Timer Management}

RPL differs from other routing protocols in timer management. LLNs network is made of low powered networks. Hence it is obvious to restrain the control plane traffic in the network. Most routing protocols use periodic control packets to keep routing tables up to date. But this is not feasible in LLNs because of resource scarcity. Hence RPL uses an adaptive timer called the "trickle timer". This timer controls the sending rate of DIO messages. Certain events are treated as inconsistencies in the network. The interval of the trickle timer increases as the network stabilizes which results in fewer DIO messages being sent in the network. If inconsistencies are detected, the nodes reset the trickle timer. So the frequency of the DIO messages depends on the stability of the network $[36,37,41]$.

\section{(iii) Security in RPL}

Security is an important concern in smart object networks. But implementation complexity and size are the major hurdles for security implementations in LLNs. Also, many deployments can utilize link-layer or other security mechanisms to meet their security requirements without requiring the use of security in RPL. Therefore, the security features are available as optional extensions in RPL [41].

\section{(iv) Frame Delivery}

Even though 802.15.4 networks commonly use mesh routing, IEEE 802.15.4-2003 specification does not define any capability. In these cases, Full Function Devices (FFDs) operates ad hoc or mesh routing protocol to populate routing tables. In such mesh scenarios, direct reachability is not required for two devices to communicate. The sender is called the "Originator" and the receiver is identified as the "Final Destination". An originator device might use other intermediate devices to forward data packets to the final destination. To achieve this kind of frame delivery using unicast, it is necessary to include the link-layer addresses of the originator and final destinations [43-45]. In the same way, if a node receives a frame with a Mesh Addressing header, it must look at the Mesh Addressing header's "Final Destination" field to determine the real destination. If it finds its address in the final destination, it receives the packet as per normal delivery. If it is not the final destination, the device then reduces the "Hops Left" field, and if the result is zero, discards the packet. Otherwise, the node consults its 
link-layer routing table, determines what the next hop towards the final destination should be, and puts that address in the destination address field of the 802.15.4 header [46]. Only "Full Function Devices" (FFDs) are expected to participate as routers in a mesh. "Reduced Function Devices" (RFDs) limit themselves to discovering FFDs and using them for all their forwarding, like how IP hosts typically use default routers to forward all their off-link traffic [43-47].

\section{MONITORING ATHLETES' THERMOREGULATION}

The major objective of this project is to train athletes' according to the climatic conditions of the race region to avoid death due to irregular thermoregulatory conditions. The climatic conditions of the race region are derived from the previously available reports and forecasts of the race season. According to the climatic conditions, athletes' are trained in the simulated environment of the race topography. Athletes' are fitted with wearable temperature sensors during training. These sensors get the athletes' body temperature readings periodically and send them to the centralized server. Data stored in the centralized server is accessible by the coach and medical personnel to review the body temperature of athletes' during training in the simulated environment. After reviewing the report, the coach and medical personnel prepare a new training schedule changing the training attributes concerning the training report of the athlete. Using IoT in monitoring the thermoregulatory process of athletes' not only trains athletes' to the climate of race topography also improves the athletes' overall progress due to personalized training schedules and reduces the number of coaches and medical professional. Also, Improves the quality of training by accurately measuring the training attributes of the athletes'.

\section{SIMULATION AND PERFORMANCE ANALYSIS}

\section{Contiki OS Cooja Simulator}

COOJA is more like an emulator as it uses real-time codes to simulate a sensor mote and a protocol. A simulated Contiki Mote in COOJA is an actual compiled code executing the Contiki system. 


\begin{tabular}{|l|l|}
\hline \multicolumn{1}{|c|}{ PARAMETERS } & \multicolumn{1}{c|}{ VALUES } \\
\hline Examined Protocol & RPL and 6LoWPAN \\
\hline Radio Medium & Unit Disk Graph Medium (UDGM) \\
\hline Ranges & TX: 50m; Interference: 60m \\
\hline MAC Layer & IEEE 802.15.4 \\
\hline Duty Cycling & $\begin{array}{l}\text { Contiki } \\
\text { Mac }\end{array}$ \\
\hline Transmission Range & $20 \mathrm{~m}$ \\
\hline Data Packet Size & 4 application layer Bytes \\
\hline Number of Nodes & $\begin{array}{l}51(\text { One root sink and 50 traffic } \\
\text { source) }\end{array}$ \\
\hline Simulation Time & 1500 seconds \\
\hline Propagation Model & Free Space \\
\hline
\end{tabular}

Table 2: Simulation parameters

\section{PERFORMANCE METRICS}

The metrics considered for the analysis of the suitability of RPL and 6LoWPAN for the above application are Packet Delivery Ratio (PDR) and Routing Protocol Overhead (RPO).

\section{Packet Delivery Ratio}

It is the ratio of the number of data packets delivered to the destined node to the number of data packets generated at the source node during the simulation time.

Routing Protocol Overhead

Routing Protocol Overhead is the overhead incurred by transmitting control packets used to maintain the Routing Topology.

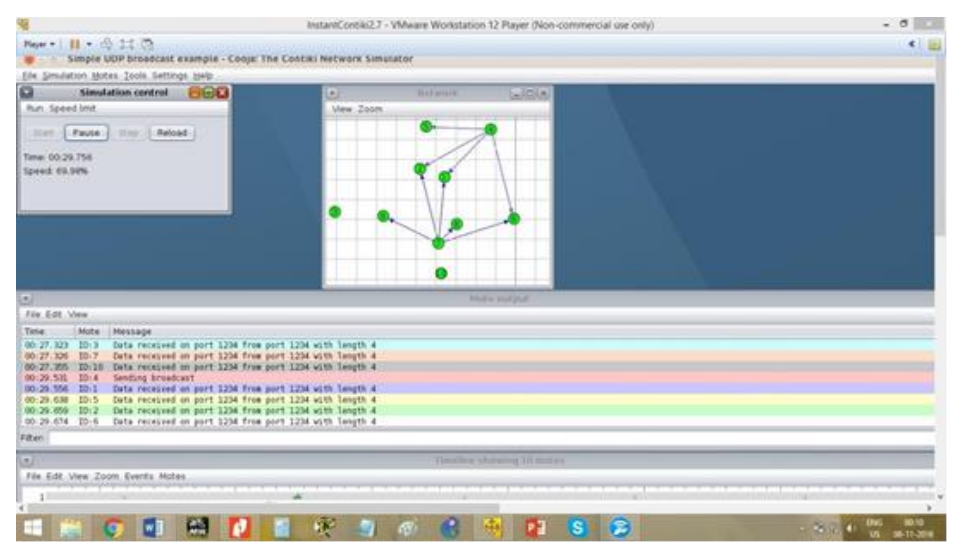

Figure 1: RPL and 6LoWPAN simulation with 51 nodes 


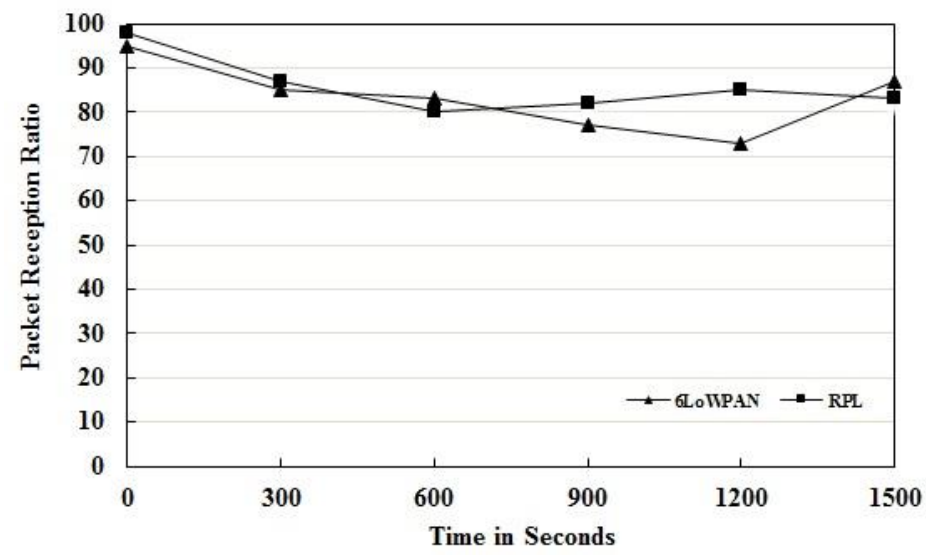

Figure 2: PRR comparison

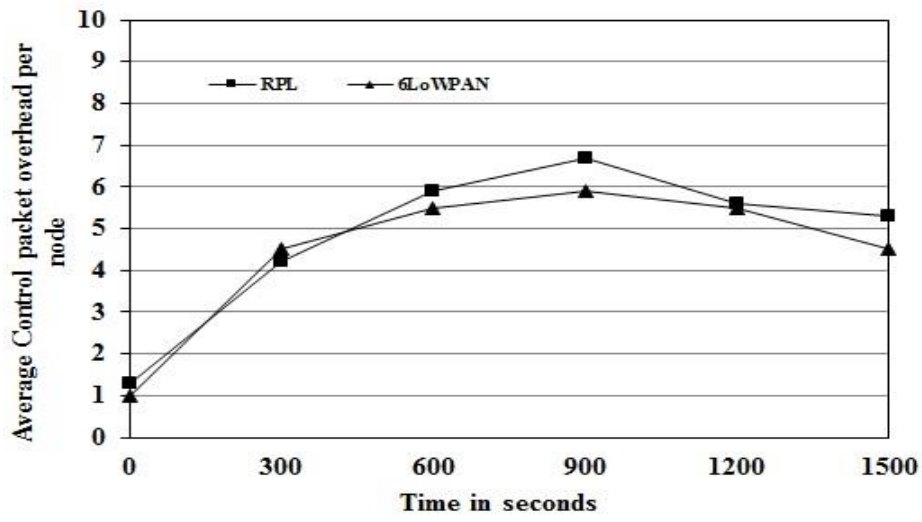

Figure 3: RPO comparison

\section{RESULTS}

As expected athletes' thermoregulation process can be precisely monitored during training and necessary training schedules can be charted down by less no. of coach aided by Internet-ofThings. Figure 2 depicts the comparison of PDR of RPL and 6LoWPAN protocols based on the simulation discussed in section 6. The above graph depicts that RPL shows better PPR if compared to 6LoWPAN. While RPL achieves 96\% PPR on average 6LoWPAN could achieve only $92 \%$ of PDR on average on the above simulation topology. Figure 3 depicts the comparison of CPO of RPL and 6LoWPAN protocols based on the simulation. The above graph depicts that RPL shows higher CPO due to frequent DIO messages if compared to 6LoWPAN which uses a lesser number of control messages. While RPL uses 7.3 control packets per node on average 6LoWPAN uses only 5.3 control packets per node on average on 
the above simulation topology. The above results show that if the node density is higher RPL works well than the 6LoWPAN protocol.

\section{CONCLUSION}

Thus using extensive analysis of the performance of 6LoWPAN and RPL IoT for healthcare applications we especially focus on monitoring athlete's thermoregulation in an enhanced manner. Also, a new technique to identify and train marathon athletes to the race topography has been proposed. In this technique, each athlete is fitted with wearable sensors in their body in the training session to monitor and analyze the thermoregulation process occurring during training. After a detailed analysis of the athletes' thermoregulation process, personal training schedules are charted down according to variation in the thermoregulation process in each athlete. This technique helps to monitor each athlete's progress personally with less number of coaches and medical professionals leading to the prevention of unexpected death of a healthy athlete.

\section{References}

[1] Tel-Aviv half Marathon race, Israel. Available Online: http://tinyurl.com/zomjuse

[2] London Marathon 2014. Available Online: http://tinyurl.com/zscvfb8

[3] Pennsylvania half marathon. Available Online: http://tinyurl.com/hcazl58.

[4] Dohr, Angelika, et al. "The Internet of Things for Ambient Assisted Living." ITNG 10 (2010):

[5] Information Society Technologies Advisory Group (ISTAG). "Revising Europe's ICT Strategy." Report from the Information Society Technologies Advisory Group (ISTAG), February 2009.

[6] Haller, Stephan, Stamatis Karnouskos, and Christoph Schroth. "The Internet of Things in an Enterprise Context." Vienna: Springer (Berlin-Heidelberg), 2008. pp. 14-28.

[7] Boyles, Justin G., Frank Seebacher, Ben Smit, and Andrew E. McKechnie. "Adaptive thermoregulation in endotherms may alter responses to climate change." Integrative and Comparative Biology (2011): icr053.

[8] Hensel H. Neural processes in thermoregulation. Physiol Rev 1973; 53: 948-1017. 
[9] Havenith G, Coenen JML, Kistemaker L, et al. Relevance of individual characteristics for human heat stress response is dependent on exercise intensity and climate type. Eur J Appl Physiol 1998; 77: 231-41.

[10] Suping Z, Guanglin M, Yanwen W, et al. Study of the relationships between weather conditions and the marathon race, and of meteorotropic effects on distance runners. Int $\mathrm{J}$ Biometeorol 1992; 36: 63-8.

[11] Trapasso LM, Cooper JD. Record performances at the Bostonmarathon: biometeorological factors. Int J Biometeorol 1989; 33: 233-7.

[12] Galloway SDR, Maughan RJ. Effects of ambient temperature on the capacity to perform prolonged cycle exercise in man. Med Sci Sports Exerc 1997; 29 (9):1240-9.

[13] Pitsiladis YP, Maughan RJ. The effects of exercise and diet manipulation on the capacity to perform prolonged exercise in the heat and in the cold in trained humans. J Physiol 1999; 517 (3): 919-30

[14] Nielsen B. Olympics in Atlanta: a fight against physics. MedSci Sports Exerc1996; 28 (6): 665-8.

[15] Armstrong LE, Hubbard RW, Jones BH, et al. Preparing Alberto Salazar for the heat of the 1984 Olympic Marathon. Phys Sportsmed 1986; 14 (3): 73-81.

[16] Adams WC, Fox RH, Fry AJ, et al. Thermoregulation during marathon running in cold, moderate, and hot environments. J Appl Physiol 1975; 38 (6): 1030-7.

[17] Beckner GL, Winsor T. Cardiovascular adaptations to prolonged physical effort. Circulation 1954; 9: 835-46.

[18] Buskirk ER, Beetham WP. Dehydration and body temperature as a result of marathon running. Med Sport 1960; 14:493-506.

[19] Cade R, Packer D, Zauner D, et al. Marathon running: physiological and chemical changes accompanying late-race functional deterioration. Eur J Appl Physiol 1992; 65 (6): 485-91

[20] Christensen CL, Ruhling RO. Thermoregulatory responses during a marathon: a case study of a women runner. Br J Sports Med 1980; 14: 131-2.

[21] Cohen I, Zimmerman AL. Changes in serum electrolyte levels during marathon running. S Afr Med J 1978; 53: 449-53 9. Kavanagh T, Shephard RJ. On the choice of fluid for the hydration of middle- aged marathon runners. Br J Sports Med 1977; 11 (1): 26-35.

[22] Kavanagh T, Shephard RH, Pandit V. Marathon running after myocardial infarction. JAMA 1974; 229 (12): 1602-5.

[23] Magazanik A, Shapiro Y, Meytes D, et al. Enzyme blood levels and water balance during a marathon race. J Appl Physiol 1974; 36 (2): 214-7. 
[24] Maron MB, Wagner JA, Horvath SM. Thermoregulatory responses during competitive marathon running. J Appl Physiol 1977; 42 (6): 909-14.

[25] Maron MB, Horvath SM, Wilkerson JE, et al. Oxygen uptake measurements during competitive marathon running. J Appl Physiol 1976; 40 (5): 836-8.

[26] Maron MB, Horvath SM, Wilkerson JE. Acute blood biochemical alterations in response to marathon running. Eur J Appl Physiol 1975; 34: 173-81.

[27] Maughan RJ. Thermoregulation in marathon competition at low ambient temperature. Int J Sports Med 1985; 6: 15-9.

[28] Maughan RJ, Leiper JB, Thompson J. Rectal temperature after marathon running.Br J Sports Med 1985; 19 (4): 192-6.

[29] Maughan RJ, Whiting PH, Davidson RJL. Estimation of plasma volume changes during marathon running. Br J Sports Med 1985; 19 (3): 138-41.

[30] Muir AL, Davidson IA, Percy-Robb IW, et al. Physiological aspects of the Edinburgh Commonwealth Games. Lancet 1972; II: 1125-8.

[31] Myhre LG, Hartung H, Nunneley SA, et al. Plasma volume changes in middle- aged male and female subjects during marathon running. J Appl Physiol 1985; 59 (2): 559-63.

[32] Myhre LG, Hartung GH, Tucker DM. Plasma volume and blood metabolites in middleaged runners during a warm-weather marathon. Eur J Appl Physiol 1982;48: 227-40.

[33] Nelson PB, Ellis D, Fu F, et al. Fluid and electrolyte balance during a cool weather marathon. Am J Sports Med 1989; 17(6): 770-2.

[34] Vasseur, J., Navneet Agarwal, Jonathan Hui, Zach Shelby, Paul Bertrand, and Cedric Chauvenet. " RPL: The IP routing protocol designed for low power and lossy networks." Internet Protocol for Smart Objects (IPSO) Alliance 36 (2011).

[35] T. Winter, P. Thubert, A. Brandt, J. Hui, R. Kelsey, RPL: IPv6 Routing Protocol for Low Power and Lossy Networks, IETF Request for Comments 6550, March 2012.

[36] Accettura, N., L. A. Grieco, G. Boggia, and P. Camarda. "Performance analysis of the RPL routing protocol." In Mechatronics (ICM), 2011 IEEE International Conference on, pp. 767-772. IEEE, 2011.

[37] Hui, J., J. P. Vasseur, D. Culler, and V. Manral. An IPv6 routing header for source routes with the routing protocol for low-power and lossy networks (RPL). No. RFC 6554. 2012.

[38] Clausen, Thomas, Ulrich Herberg, and Matthias Philipp. "A critical evaluation of the IPv6 routing protocol for low power and lossy networks (RPL)." In 2011 IEEE 7th International Conference on Wireless and Mobile Computing, Networking and Communications (WiMob), pp. 365-372. IEEE, 2011. 
[39] Ancillotti, Emilio, Raffaele Bruno, and Marco Conti. "RPL routing protocol in advanced metering infrastructures: an analysis of the unreliability problems." In Sustainable Internet and ICT for Sustainability (SustainIT), 2012, pp. 1-10. IEEE,2012

[40] Ko, JeongGil, Andreas Terzis, Stephen Dawson-Haggerty, David E. Culler, Jonathan W. Hui, and Philip Levis. "Connecting low-power and lossy networks to the internet." IEEE Communications Magazine 49, no. 4 (2011): 96-101.

[41] Sheng, Zhengguo, Shusen Yang, Yifan Yu, Athanasios V. Vasilakos, Julie A. McCann, and Kin K. Leung. "A survey on the ietf protocol suite for the internet of things: Standards, challenges, and opportunities." IEEE Wireless Communications 20, no. 6 (2013): 91-98.

[42] Granjal, Jorge, Edmundo Monteiro, and Jorge Sá Silva. "Security for the internet of things: a survey of existing protocols and open research issues." IEEE Communications Surveys \& Tutorials 17, no. 3 (2015): 1294-1312.

[43] Montenegro, Gabriel, Nandakishore Kushalnagar, Jonathan Hui, and DavidCuller. Transmission of IPv6 packets over IEEE 802.15. 4 networks. No. RFC 4944. 2007.

[44] Mulligan, Geoff. "The 6LoWPAN architecture." In Proceedings of the 4th workshop on Embedded networked sensors, pp. 78-82. ACM, 2007.

[45] Kushalnagar, N., Montenegro, G. and Schumacher, C., 2007. IPv6 over low- power wireless personal area networks (6LoWPANs): overview, assumptions, problem statement, and goals.

[46] Oliveira, Luís ML, Amaro F. De Sousa, and Joel JPC Rodrigues. "Routing and mobility approaches in IPv6 over LoWPAN mesh networks." International Journal of Communication Systems 24, no. 11 (2011): 1445-1466.

[47] Da Xu, L., He, W. and Li, S., 2014. Internet of things in industries: A survey. IEEE Transactions on Industrial Informatics, 10(4), pp.2233-2243. 